\title{
High-resolution x-ray crystal spectrometer/polarimeter at torus experiment for technology oriented research-94
}

\author{
J. Weinheimer, I. Ahmad, O. Herzog, and H.-J. Kunze \\ Institut für Experimentalphysik V, Ruhr-Universität Bochum, D-44780 Bochum, Germany \\ G. Bertschinger ${ }^{\text {a) }}$ and W. Biel \\ Institut für Plasmaphysik, Forschungzentrum Juelich GmbH, Assoc. Euratom-KFA, D-52425 Juelich, \\ Germany, Trilateral Euregio Cluster \\ G. Borchert \\ Institut für Kernphysik, Forschungzentrum Juelich GmbH, D-52425 Juelich, Germany \\ M. Bitter \\ Princeton Plasma Physics Laboratory, Princeton University, Princeton, New Jersey 08543
}

(Received 1 November 2000; accepted for publication 19 March 2001)

\begin{abstract}
A high-resolution x-ray polarimeter has been installed at the torus experiment for technology oriented research (TEXTOR-94). The instrument consists of two spectrometers in Johann geometry, utilizing the dependence of Bragg reflection on the polarization. The dispersion plane of the so-called horizontal spectrometer coincides with the midplane of the tokamak, whereas the dispersion plane of the so-called vertical spectrometer lies perpendicular to the midplane of the tokamak. Both spectrometers operate with cylindrically bent quartz crystals of dimensions $153 \mathrm{~mm} \times 38 \mathrm{~mm} \times 0.7 \mathrm{~mm}$ with a $2 d$ spacing of $4.9130 \AA$. The radii of curvature of the horizontal and vertical spectrometer crystals are $3820 \mathrm{~mm}$ and $4630 \mathrm{~mm}$, respectively. The detectors are multiwire proportional counters having a large entrance window of $180 \mathrm{~mm} \times 90 \mathrm{~mm}$, with a high count rate capability of up to $2.5 \times 10^{5}$ photons/s, and a spatial resolution of $0.4-0.65 \mathrm{~mm}$, depending on the count rate. The unique data acquisition system makes it possible to record up to 8192 spectra per TEXTOR-94 discharge to study fast events and impurity transport. The instruments are sensitive to spurious amounts of argon in hydrogen or deuterium discharges and aim for $\mathrm{x}$-ray polarization measurements and other core plasma diagnostics like electron temperature, ion temperature, plasma rotation, impurity transport, and relative abundance of $\mathrm{Ar}^{15+} / \mathrm{Ar}^{16+}$. The first measurements of toroidal rotation speed, ion and electron temperatures show a very good agreement with charge exchange recombination spectroscopy (XRS) and electron cyclotron emission (ECE) diagnostics available at TEXTOR-94. The measured values of the ratio $\mathrm{Ar}^{15+} / \mathrm{Ar}^{16+}$ are higher than that predicted from coronal equilibrium. The ratio depends strongly upon the electron density both for ohmic and neutral beam injection cases. This suggests that the charge state distribution is also dependent on diffusion and charge exchange processes in the plasmas. () 2001 American Institute of Physics. [DOI: 10.1063/1.1370558]
\end{abstract}

\section{INTRODUCTION}

A high-resolution $\mathrm{x}$-ray polarimeter has recently been installed at the torus experiment for technology oriented research (TEXTOR-94) to measure the polarization of $\mathrm{x}$-ray lines. ${ }^{1}$ The polarization of $\mathrm{x}$-ray lines provides information on deviations of the electron energy distribution from a Maxwellian, ${ }^{2}$ which can, for instance, be produced in plasmas with auxiliary heating by electron cyclotron waves. The polarimeter was built in collaboration with the Princeton Plasma Physics Laboratory and consists of two highresolution x-ray crystal spectrometers with mutually perpendicular dispersion planes. Both instruments record spectra of helium-like argon (ArXVII) in the narrow wavelength region of 3.9-4.0 $\AA$. This wavelength range encompasses the $1 s^{2}\left({ }^{1} S_{0}\right)-1 s 2 p\left({ }^{1} P_{1}\right)$ resonance line, the associated forbid-

${ }^{\text {a)} E l e c t r o n i c ~ m a i l: ~ g . b e r t s c h i n g e r @ f z-j u e l i c h . d e ~}$ den and intercombination lines as well as lithium-like satellites (Fig. 1). The spectra provide measurements of the central ion temperature, ${ }^{3-5}$ central electron temperature, ${ }^{6}$ central toroidal rotation, and distribution of the highly ionized charge states of argon, which in combination with other diagnostics can be used to study impurity transport. ${ }^{7}$

Spectra of helium-like argon have previously been observed at the Tokamak Fontenay aux Roses (TFR) $)^{8,9}$ and at the Alto Campo Torus (ALCATOR) tokamaks, where they are being used for the routine diagnostic purposes. ${ }^{10}$ The experiments at TFR were performed with a helium-filled Johann crystal spectrometer, while the measurements at ALCATOR were performed with von Hamós type spectrometers.

The Johann geometry, which is the most commonly used at tokamaks has also been adopted for the spectrometers at TEXTOR-94. The Johann configuration has the advantage that the whole spectral range is recorded simultaneously and 


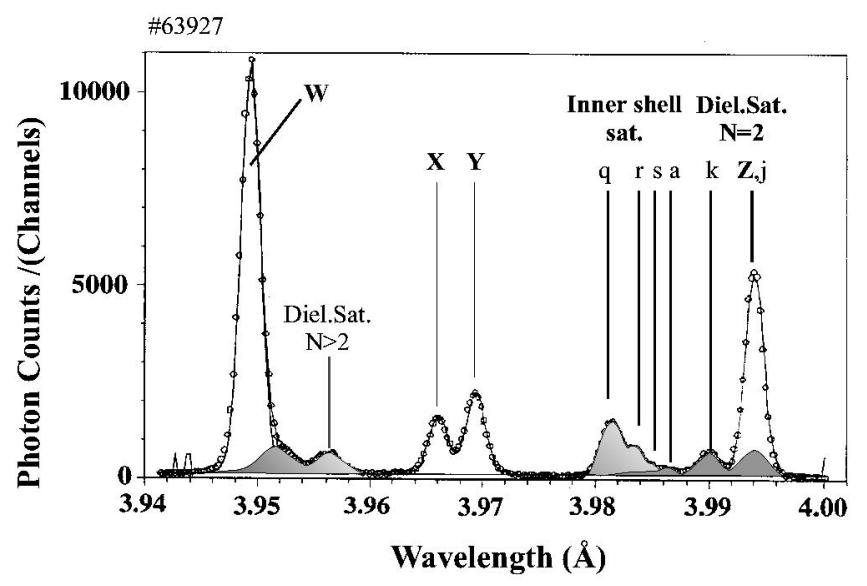

\begin{tabular}{|c|c|c|}
\hline Key & Transition & Vavelength $(\AA)^{*}$ \\
\hline $\mathbf{W}$ & $1 \mathrm{~s}^{21} \mathrm{~S}_{0}-1 \mathrm{~s} 2 \mathrm{p}^{1} \mathrm{P}_{1}$ & 3.9494 \\
\hline $\mathbf{X}$ & $1 s^{21} S_{0}-1 s 2 p^{3} P_{2}$ & 3.9661 \\
\hline $\mathbf{Y}$ & $1 s^{21} S_{0}-1 s 2 p^{3} P_{1}$ & 3.9696 \\
\hline$q$ & $1 s^{2} 2 s^{2} \mathrm{~S}_{1 / 2}-1 \mathrm{~s} 2 \mathrm{~s} 2 \mathrm{p}\left({ }^{1} \mathrm{P}\right){ }^{2} \mathrm{P}_{3 / 2}$ & 3.9815 \\
\hline$r$ & $1 s^{2} 2 s^{2} S_{1 / 2}-1 s 2 s 2 p\left({ }^{1} P\right){ }^{2} P_{1 / 2}$ & 3.9836 \\
\hline $\mathrm{a}$ & $1 \mathrm{~s}^{2} 2 \mathrm{p}^{2} \mathrm{P}_{3 / 2}-1 \mathrm{~s} 2 \mathrm{p}^{2} \mathrm{P}_{3 / 2}$ & 3.9860 \\
\hline $\mathrm{k}$ & $1 s^{2} 2 p^{2} P_{1 / 2}-1 s 2 p^{2} D_{3 / 2}$ & 3.9901 \\
\hline $\mathrm{j}$ & $1 \mathrm{~s}^{2} 2 \mathrm{p}^{2} \mathrm{P}_{3 / 2}-1 \mathrm{~s} 2 \mathrm{p}^{2} \mathrm{D}_{5 / 2}$ & 3.9941 \\
\hline $\mathbf{Z}$ & $1 s^{21} S_{0}-1 s 2 s^{3} S_{1}$ & 3.9944 \\
\hline
\end{tabular}

* Theory by Vainshtein and Safranova

FIG. 1. Experimental ArXVII spectrum with corresponding satellites obtained on TEXTOR-94. Lines originating from ArXVI and ArXVIII are also present. The shaded regions illustrate the contributions from the dielectronic satellites to the spectrum.

that all the photons, which contribute to a spectral line are reflected from the entire crystal. Therefore, one obtains high throughput as well as reliable line intensity ratios, since variations of the reflectivity along the crystal affect each spectrum line in the same way.

The spectrometers at TEXTOR-94 have been designed as vacuum instruments, to eliminate the attenuation of $\mathrm{x}$ rays in air and to enhance the sensitivity to spurious amounts of argon in the plasma. This assures a consistent performance, since changes of the spectrometer throughput, which otherwise might result from variations in the purity of a helium atmosphere, have been avoided. A unique data acquisition system makes it possible to record up to 8192 spectra per discharge and to study fast events. Both spectrometers operate presently with cylindrically bent crystals. However, the so-called horizontal spectrometer will soon be equipped with a spherically bent crystal and a two-dimensional (2D) position-sensitive detector. The spherically bent crystal will provide spatial resolution in a direction perpendicular to the horizontal midplane of TEXTOR-94, so that it will be possible to obtain radial profiles and gradients of plasma parameters with only one crystal. ${ }^{11}$ These new features and the possibility to perform measurements of the polarization of

\section{Coronal Equilibrium}

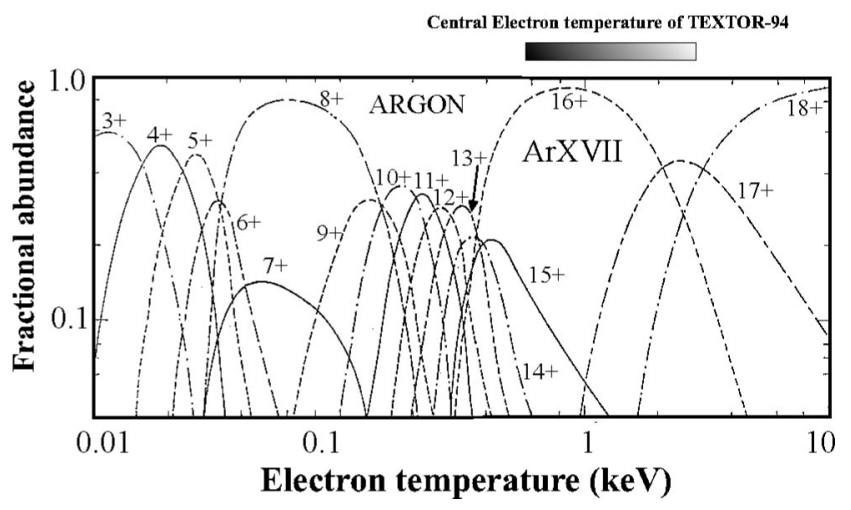

FIG. 2. Coronal equilibrium of charge states for argon. ArXVII is present over the whole range of electron temperatures accessible to TEXTOR-94.

$\mathrm{x}$-ray lines will advance the state of the art of $\mathrm{x}$-ray spectroscopy at tokamaks.

TEXTOR-94 is a medium-size tokamak experiment, which operates with magnetic fields of up to $2.8 \mathrm{~T}$ and currents of up to $700 \mathrm{kA}$. The major and minor radii of the plasma torus are of 1.75 and $0.46 \mathrm{~m}$, respectively. In addition to the ohmic heating of about $0.5 \mathrm{MW}$, which is obtained from the plasma current, auxiliary heating is provided by the injection of neutral hydrogen beams with power up to $4 \mathrm{MW}$ and by ion cyclotron waves resonance heating with a power of up to $4.5 \mathrm{MW}$. Further auxiliary heating system by electron cyclotron waves has recently been installed with power up to $500 \mathrm{~kW}$. The electron temperatures and electron densities in TEXTOR-94 are in the range from 0.5 to $2.5 \mathrm{keV}$ and from 0.1 to $1 \times 10^{14} \mathrm{~cm}^{-3}$, respectively.

The experiments at TEXTOR-94 are dedicated to the study of plasma-wall interactions, impurity transport, and magnetohydrodynamic instabilities, which affect the confinement in the core plasma. To address these topics TEXTOR-94 is equipped with a large number of diagnostics, a detailed description can be found in Ref. 1.

The high-resolution x-ray crystal spectrometers supplement the core diagnostics at TEXTOR-94 in an important way by extending the diagnostic of the central ion temperature and central plasma rotation to plasmas with pure ohmic heating and $\mathrm{rf}$ heating. They are also routinely used for the diagnostics of plasmas with neutral-beam injection. Since ArXVII is the dominant charge state for electron temperatures in the range from 0.5 to $3.5 \mathrm{keV}$-which corresponds to the range of central electron temperatures in TEXTOR94- the crystal spectrometers can provide data for the entire range of operating conditions (Fig. 2). Another experimental advantage results from the fact that the concentration of argon in the plasma can easily be controlled by gas puffing. The knowledge gained by studying argon in TEXTOR-94 may be valuable for larger tokamaks as well, since argon puffing is being applied to larger tokamaks ${ }^{12}$ to cool the plasma edge by radiation in order to obtain a homogeneous release of energy to the plasma-facing components.

The crystal spectrometers at TEXTOR-94 are also well suited for the study of fast events like sawtooth oscillations, ${ }^{1}$ 
since they provide simultaneous data on a number of crucial plasma parameters such as ion temperature, plasma rotation, electron temperature, and charge state distribution. In addition to these diagnostic applications, the spectrometers are used to address atomic physics questions and to investigate atomic processes like charge exchange, which affect the ionization equilibrium. ${ }^{13}$

To date, in addition to the standard measurements of ion temperature, electron temperature, and toroidal rotation, the system has been used to study polarization in ohmic discharges, the time behavior of plasma parameters during sawtooth oscillations, the effects of charge exchange recombination of ArXVII with neutral hydrogen, and impurity transport. ${ }^{1,2,13}$ For the future, it is planned to develop one of the instruments into an imaging X-ray crystal spectrometer and to address a number of research topics, which include the search for polarization during sawtooth oscillations, a comparison of theoretical excitation rates with experimental data from tokamak plasmas, a study of the plasma rotation as a function of plasma parameters, and an investigation of polarization effects in plasmas with electron cyclotron heating which has recently been installed at TEXTOR-94.

\section{CHARACTERISTICS OF THE INSTRUMENT}

The requirements for the $\mathrm{x}$-ray crystal spectrometers at TEXTOR-94 are: high spectral resolution, high throughput, high time resolution, and high sensitivity. High spectral resolution is needed for Doppler measurements of the ion temperature and plasma rotation, and high throughput is required for the study of fast events. Finally, high sensitivity is desired to perform measurements with small nonperturbing amounts of argon in the plasma.

\section{A. Experimental arrangement and instrumental layout}

The X-ray polarimeter at TEXTOR-94 consists of two Johann crystal spectrometers, which operate at Bragg angles of $54^{\circ}$. This angle is close to the Brewster angle, for which only one state of polarization is reflected by the crystal. The dispersion plane of the first so-called horizontal spectrometer coincides with the horizontal midplane of TEXTOR-94, while the dispersion plane of the other (vertical) spectrometer is perpendicular to the horizontal midplane. Both instruments are presently equipped with cylindrically bent crystals and 1D position-sensitive detectors placed on the Rowland circle perpendicular to the incident $\mathrm{x}$ rays. The experimental arrangement is shown in Fig. 3.

The crystals used in the spectrometers are 110 quartz crystals with a $2 d$ spacing of $4.9130 \AA$ and they are of dimensions $153 \mathrm{~mm} \times 38 \mathrm{~mm} \times 0.7 \mathrm{~mm}$. The radii of curvature of the crystals are 3820 and $4630 \mathrm{~mm}$ and the distances between the crystals and detectors are 3050 and $3736 \mathrm{~mm}$, for the horizontal and vertical spectrometer, respectively. The detectors are multiwire proportional counters from the former x-ray crystal spectrometers at the Tokamak Fusion Test Reactor in Princeton. ${ }^{14,15}$ These detectors have a large entrance window of $180 \mathrm{~mm} \times 90 \mathrm{~mm}$, a high count rate capability of up to $2.5 \times 10^{5}$ photons/s, and a spatial resolution of 0.4-0.65 mm, depending on the count rate.

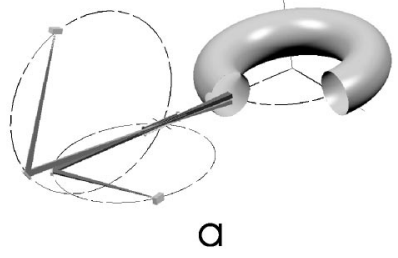

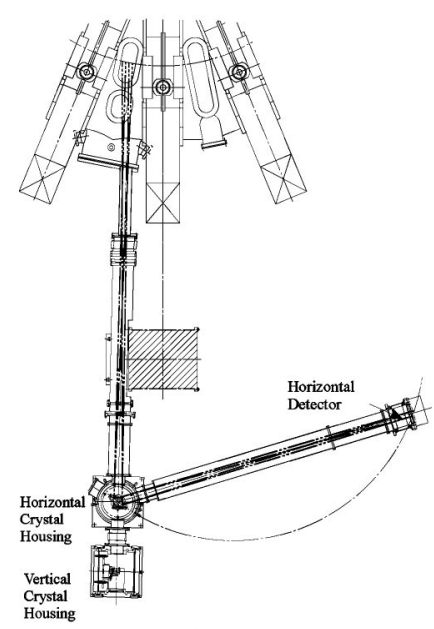

b

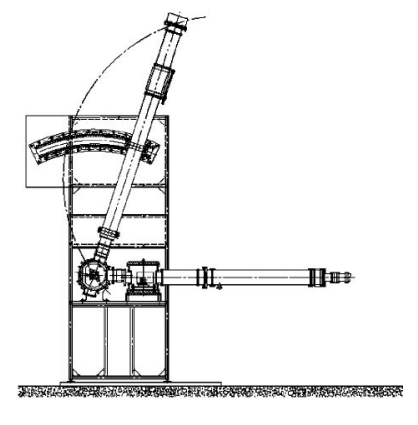

C
FIG. 3. (a) The X-ray polarimeter at TEXTOR-94 consists of two conventional $\mathrm{x}$-ray spectrometers in the Johann configuration with Bragg angles of $54^{\circ}$ viewing the plasma from the same line of sight. (b) The first spectrometer (horizontal) has the dispersion plane coincident to the midplane of the tokamak and (c) the second spectrometer (vertical) has the dispersion plane perpendicular to the midplane of the tokamak. The polarimeter measures $\mathrm{x}$-ray radiation in two states of linear polarization with high spectral resolution.

The crystals of the horizontal and the vertical x-ray spectrometers are located at distances of 4300 and $5035 \mathrm{~mm}$ from the center of the vacuum vessel of TEXTOR-94, respectively. The spectrum of interest, which extends from the resonance line w: $1 s^{2}\left({ }^{1} S_{0}\right)-1 s 2 p\left({ }^{1} P_{1}\right)$ at $3.9494 \AA$ to the forbidden line $\mathbf{z}: 1 s^{2}\left({ }^{1} S_{0}\right)-1 s 2 s\left({ }^{3} S_{0}\right)$ at $3.9944 \AA$, can be observed with an unrestricted view into the plasma. The distances between the positions of the lines $\mathbf{w}$ and $\mathbf{z}$ on the detector are 47 and $58 \mathrm{~mm}$, for the horizontal and vertical spectrometers, respectively. The central line of sight of the spectrometers are at an angle $\alpha_{0}=10^{\circ}$ with respect to the major radius of the plasma torus, so that it is possible to observe a component of about $17 \%$ of the toroidal plasma velocity from the Doppler shift of the spectral lines [Fig. 3(b)]. In the case of the horizontal spectrometer, the angle $\alpha$ between a line of sight and a major radius vector varies from $11.2^{\circ}$ for the line $\mathbf{w}$ to $9.0^{\circ}$ for the line $\mathbf{z}$, whereas in the case of the vertical spectrometer $\alpha=\alpha_{0}$ for all spectral lines.

The choice of the crystals and their radii of curvature were determined by the aforementioned requirements and the constraints with respect to the available diagnostic space at TEXTOR-94. The distances from the crystals to the detectors were chosen such that the $\mathbf{z}$ line would be in focus on the Rowland circle.

\section{B. Throughput and sensitivity of the polarimeter}

The polarimeter has been designed as a vacuum instrument to eliminate attenuation of the $3 \mathrm{keV} x$ rays by air 
along the long x-ray path in the spectrometers. The spectrometer vacuum is separated from the main vacuum of the machine by a $25 \mu \mathrm{m}$ thick, $100 \mathrm{~mm}$ wide, and $160 \mathrm{~mm}$ long beryllium window. The other boundaries of the polarimeter vacuum vessel are two $25 \mu \mathrm{m}$ thick polyester foils, one placed in front of each detector. The total transmission of the system is about $15 \%$. This results from the $25 \mu \mathrm{m}$ thick beryllium foil (91\% transmission) and its metal grid support structure (66\% transmission), which separate the spectrometer from the tokamak, a $100 \mu \mathrm{m}$ thick beryllium entrance window to the detector ( $70 \%$ transmission), a $25 \mu \mathrm{m}$ polyester foil at the detector arm exit window (65\% transmission), and a $35 \mathrm{~mm}$ air gap (54\% transmission) in front of the detector. The absolute throughput of the system is also determined by the apertures of the crystals, which are described in Sec. II F.

With the present configuration of the spectrometers, it is possible to obtain count rates of $2.5 \times 10^{5}$ photons/s, corresponding to the maximum count rate for the detectors, with small nonperturbing argon gas puffs of $1.3 \mathrm{mbar} 1 / \mathrm{s}$. If required, the sensitivity of the spectrometers can be enhanced by a factor of two by filling the $35 \mathrm{~mm}$ wide air gap between the polyester foil and the entrance window of the detector with helium. This will increase the total transmission to $27 \%$.

\section{Apertures}

The exposed areas of the crystals and the entrance windows of the detectors were reduced in order to avoid vignetting of the field of view by the diagnostic port and other components in the system and to ensure that the throughput of the two spectrometers were about equal for this choice of apertures. This was important in order to obtain the maximum count rates in both spectrometers without exceeding the count rate limits of the detectors. The optimization of the polarimeter resulted in an exposed area of $70 \mathrm{~mm} \times 8.5 \mathrm{~mm}$ and $30 \times 16 \mathrm{~mm}$ for the horizontal and vertical crystals, respectively, and apertures of $18 \mathrm{~mm} \times 180 \mathrm{~mm}$ on the entrance windows of the detectors. The reduction in height of the entrance window of a detector also minimizes the loss of spectral resolution, which results from the curvature of the spectral lines.

\section{Crystal bending}

The two $153 \mathrm{~mm} \times 38 \mathrm{~mm} \times 0.7 \mathrm{~mm}$ large quartz crystals used for the horizontal and vertical spectrometers have been cylindrically bent to a radius of curvature of 3820 and 4630 $\mathrm{mm}$, respectively, with the help of a four-bar bending jig. The crystal curvature was tested optically by means of the setup shown in Fig. 4. For this purpose, a $1000 \AA$ thick reflecting layer of aluminum was deposited on the front surface of each crystal. The whole front surface of the crystal was illuminated in near-normal incidence by a point source, which was obtained by focusing a laser beam onto a pinhole. The distance between the pinhole and the crystal corresponded to the desired radius of curvature. The crystal was bent until a rough focus was formed by the reflected light on a screen positioned near the pinhole. To facilitate the bending, a mirror was then placed nearly equidistant from the

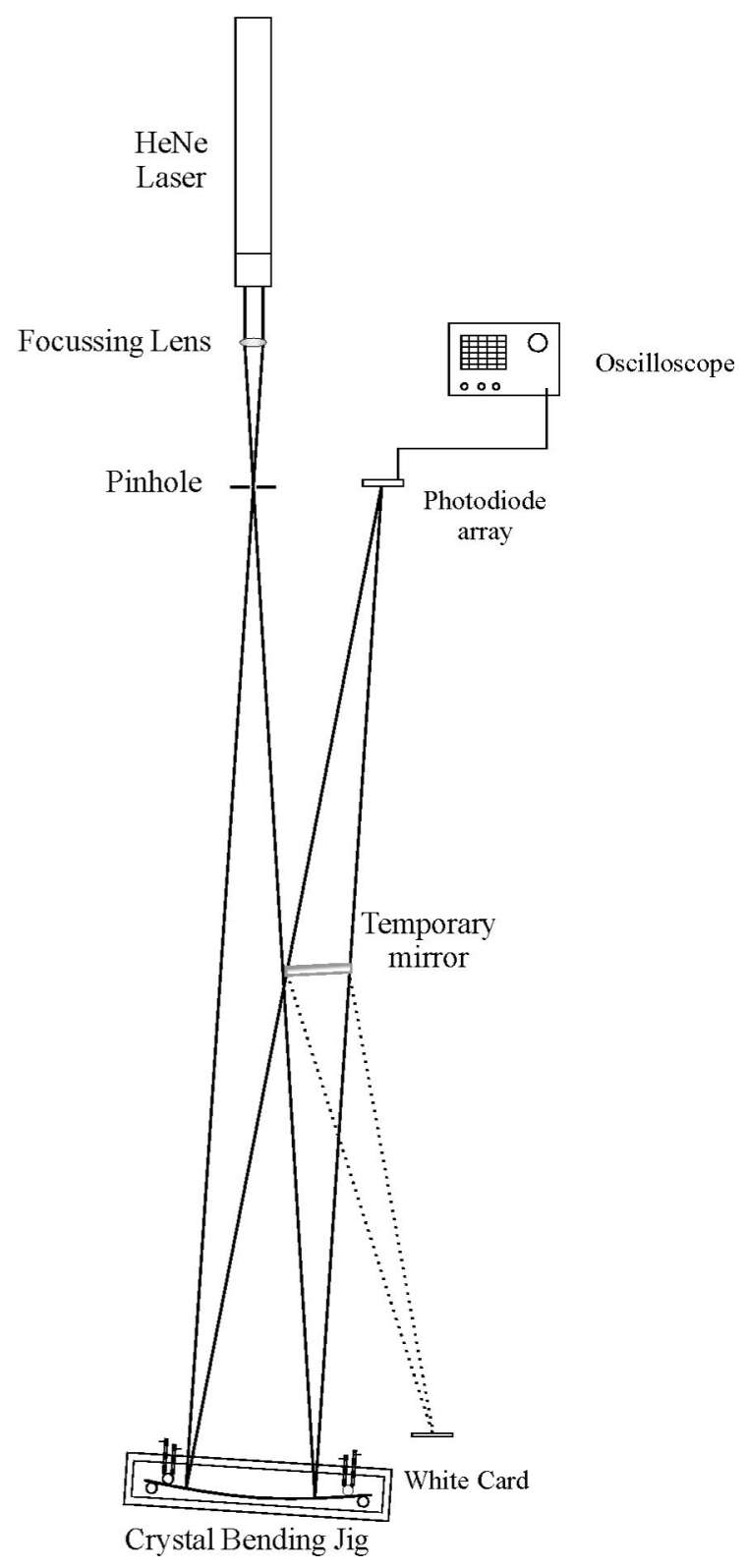

FIG. 4. Procedure for bending the spectrometer crystals and determining the associated bending errors. A $\mathrm{He}-\mathrm{Ne}$ laser was focused on a pinhole to produce a point-like source. The source was then used to illuminate the crystal placed at a distance corresponding to the radius of curvature of the crystal. The reflected light was refocused at the laser, where a photodiode measured the intensity distribution to determine the quality of the bending. A mirror was placed temporarily between the laser and crystal to reflect the light back to a white card placed next to the crystal holder to make the initial bending easier.

laser and crystal in the reflected beam, and a screen was placed next to the crystal for a close observation of the focus while the screws in the bending jig were turned. The crystal was bent until a narrow homogenous line focus appeared on the screen. The mirror was then removed and a photodiode array was placed next to the pinhole. Fine adjustments of the crystal curvature were then made to get the best focus for the crystal on the array. The resulting shape of the images measured by the photodiode array were found to be Gaussian with a full width at half maximum (FWHM) of 0.1 and 0.28 $\mathrm{mm}$ for the horizontal and vertical system, respectively. 


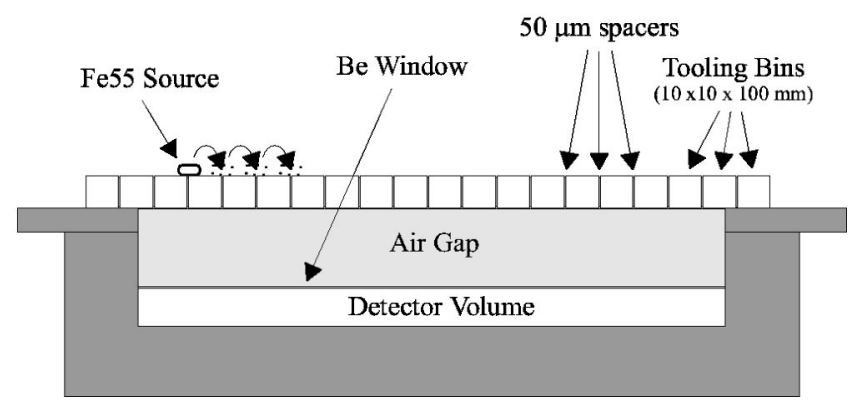

FIG. 5. The resolution and linearity of the detectors were measured using an array of $50 \mu \mathrm{m}$ collimators and a Fe 55 source. The resulting lines were fit using Gaussian profiles to determine the resolution and central position of each line. The spacing between the lines was compared with the well established positions of the slits to determine the nonlinearity of the detectors.

\section{E. Detector calibration}

The detectors used in the spectrometers are 1D multiwire proportional counters ${ }^{14,15}$ having a large entrance window, which is covered by a $180 \mathrm{~mm} \times 90 \mathrm{~mm} \times 0.1 \mathrm{~mm}$ beryllium foil. The sensitive volume of each detector is $180 \mathrm{~mm}$ $\times 90 \mathrm{~mm} \times 12 \mathrm{~mm}$. The detectors have been partially covered to reduce high x-ray flux and to avoid vignetting of the observed spectrum (see Sec. IIF). The exposed area of the entrance window is only $18 \mathrm{~mm} \times 180 \mathrm{~mm}$. The detectors operate with a mixture of $90 \% \mathrm{Kr}$ and $10 \% \mathrm{CO}_{2}$, which is used as a quenching gas. The detection efficiency for the ArXVII lines near $4 \AA$ is $99 \%$. The spatial resolution and linearity of the detectors were determined by using a Fe 55 source with an array of $50 \mu \mathrm{m}$ wide collimating slits (Fig. 5). The resulting lines were then fitted using a Gaussian line shape. The spatial resolution was found to be $0.65 \mathrm{~mm}$, when the detectors were optimized for a high count rate of 250000 photons/s. Beyond this count rate, the response of the detectors became nonlinear due to multiphoton events and spacecharge effects in the detector. A better spatial resolution of $0.4 \mathrm{~mm}$ was obtained by increasing the high voltage on the detectors, since the spatial resolution is a function of the high voltage applied to the anode wires, but this resulted in a lowering of the maximum attainable count rate. The spatial linearity of the detectors was measured by moving the Fe 55 source over the accurately spaced collimating slits. A linear fit was made to the data for the central region of the detector from 50 to $150 \mathrm{~mm}$. Figure 6 shows the deviation of the measurements from the linear fit in terms of channel widths for the horizontal detector. The maximum deviation from the linear fit over the central region from $50-150 \mathrm{~mm}$ is $0.1 \mathrm{~mm}$. For the argon spectrum, which has a dispersion of approximately $1 \times 10^{-3} \AA / \mathrm{mm}$, this corresponds to a wavelength precision $1 \times 10^{-4} \AA$. If more accuracy in the wavelengths is required, details of the detector linearity must be taken into account.

\section{F. Data acquisition system}

The data acquisition system employs two time-to-digital converters (Le Croy 4604) with symmetric start and stop inputs, which require no external delay line minimizing the conversion time. Large 16 megabyte histogramming memories (Struck STR521 HMU) make it possible to record up to

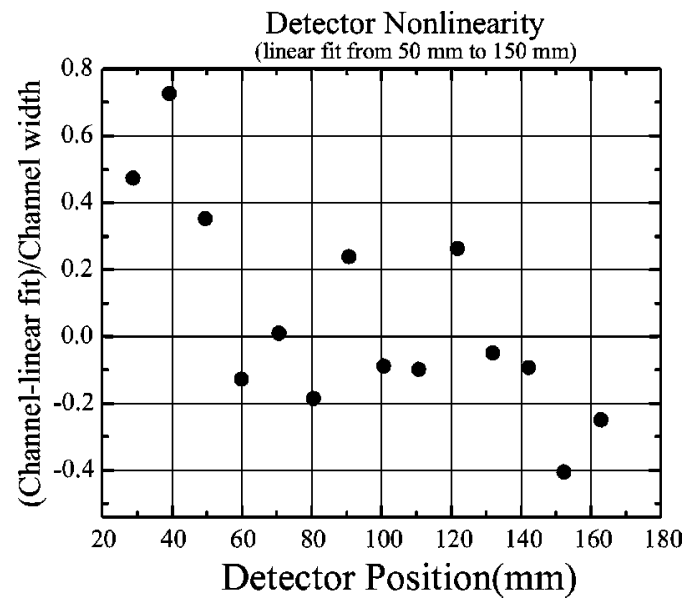

FIG. 6. The measured nonlinearity of the multiwire proportional counter. The maximum deviation from a linear fit for $50-150 \mathrm{~mm}$ on the detector is 0.4 channels, corresponding to a spectral position deviation of $1 \times 10^{-4} \AA$.

8192 spectra per discharge and to cover the full discharge with a time resolution of $0.5 \mathrm{~ms}$ per spectrum.

\section{G. Spectral resolution}

The overall resolution of the spectrometers is given by

$$
\lambda / \Delta \lambda=\tan \theta / \Delta \theta=\tan \theta^{*} R \sin \theta / \Delta X,
$$

where $\theta$ is the Bragg angle, $R$ is the radius of curvature of the crystal, and $\Delta X$ is the instrumental width. The instrumental width results from the detector resolution $X_{D}$, the crystal bending error $X_{C}$, the Johann error $X_{J}$, the rocking curve of the crystal $X_{\mathrm{RC}}$, and from the defocusing resulting from the detector not being on the Rowland circle.

The detector resolution $X_{D}$ was 0.65 or $0.4 \mathrm{~mm}$, depending on the high voltage, which was applied to the anode wires, as already mentioned in Sec. II D. The crystal bending errors $X_{C}$ were 0.1 and $0.28 \mathrm{~mm}$ for the crystals in the horizontal and vertical spectrometers, respectively, as described in Sec. II B. The rocking curve of the crystal was calculated using the dynamical theory with equal contributions from each state of polarization by means of the ray tracing program XRT developed by Borchert. ${ }^{16}$ Special attention has been paid to calculating the rocking curve, since the rocking curve of a crystal may be changed in the bending process due to elastic deformations of the crystal lattice. The changes to the rocking curve depend on the radius of curvature of the crystal and on the x-ray energy. Low-energy $\mathrm{x}$ rays do not penetrate deep into the crystal lattice and the distortions of the rocking curve seen with low-energy $\mathrm{x}$ rays are smaller than those seen with high-energy $x$ rays. The critical radius of curvature, at which distortions of the rocking curve appear, is therefore smaller for low-energy $\mathrm{x}$ rays than for highenergy $x$ rays. On the other hand, one has to keep in mind that low-energy $\mathrm{x}$ rays are much more sensitive to distortions of the crystal surface. A systematic study of these effects has been published by Uschmann et al. ${ }^{17}$ Once the rocking curve was obtained, the contributions of the Johann error and defocusing errors were determined by ray tracing using all relevant geometrical, experimental, and crystal parameters as well as the detector resolution. A Monte Carlo method was 


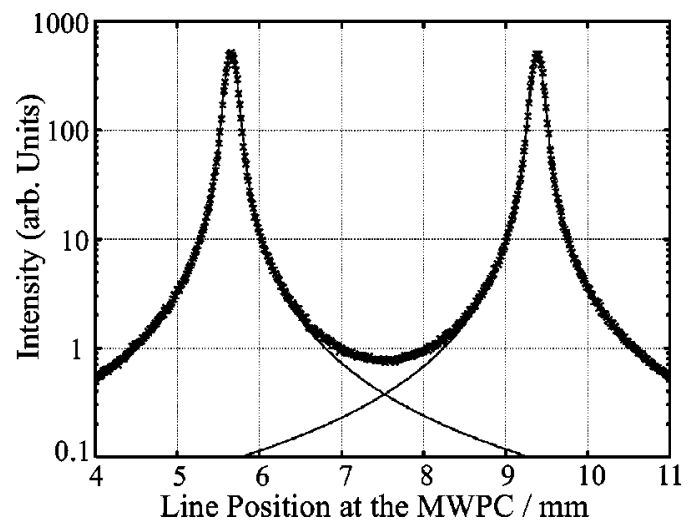

FIG. 7. The instrumental profile for the horizontal spectrometer. The detector was placed on the Rowland circle in such a way that midway between $x$ and $y$ lines is located on the Rowland circle. Solid lines indicate Voigt profile approximated to the data. The rocking curve of the crystal was calculated using dynamical theory taking into account the deformation of the crystal due to bending. Detector resolution and bending errors were omitted.

used to avoid multidimensional integration. The complete instrumental profile was obtained by convoluting the results of the ray tracing with the bending error. The resulting instrumental functions were found to be well approximated by Voigt functions. Figure 7 shows the resulting instrumental function for the horizontal detector, located on the Rowland circle in such a way that midway between $x$ and $y$ lines lies on the Rowland circle. The figure also shows a fit of the profile with Voigt profiles. Here, the detector resolution was taken to be $0 \mathrm{~mm}$. Figure 8 shows FWHM of the Lorentzian and Gaussian contributions to the complete instrumental profile as a function of position on the horizontal detector.

The spectral resolution was calculated for both spectrometers for the line on the Rowland circle. The resulting spectral resolution for the horizontal system is $\lambda / \Delta \lambda$ $=6000$ when the detector is optimized for count rate and $\lambda / \Delta \lambda=9000$ when the detector is optimized for resolution. The spectral resolution for the vertical system is $\lambda / \Delta \lambda$ $=6800$ when the detector is optimized for count rate and $\lambda / \Delta \lambda=9500$ when the detector is optimized for resolution. For a least squares fit of the experimental spectral lines, a

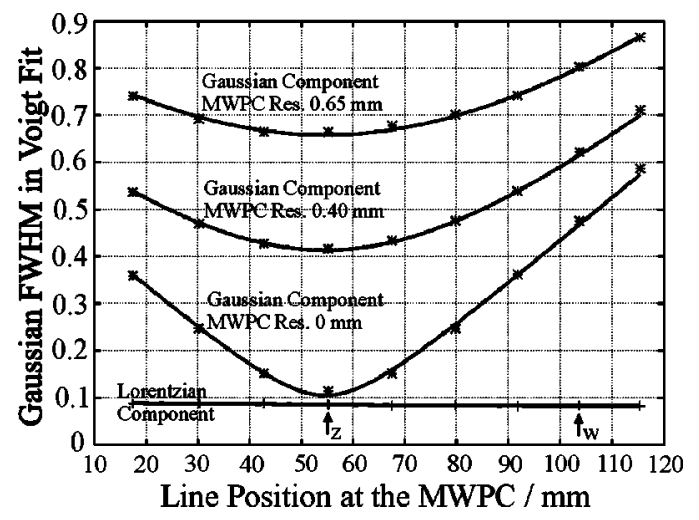

FIG. 8. The contribution of the Guassian and Lorentzian components to the instrumental width as a function of the horizontal detector position. The instrumental profile was determined by ray tracing discrete wavelengths using the XRT program developed by Borchert et al. A Voigt function was then fit to the resulting profiles to provide an easily representable instrumental function.
TABLE I. Beam power, line integrated electron density, and central electron temperature for the discharges used in the comparison of plasma rotation and ion temperature measurements made by $\mathrm{x}$-ray spectroscopy and charge exchange spectroscopy.

\begin{tabular}{ccccc}
\hline \hline $\begin{array}{c}\text { Shot } \\
\text { number }\end{array}$ & $\begin{array}{c}\text { Beam } \\
1(\mathrm{MW})\end{array}$ & $\begin{array}{c}\text { Beam } \\
2(\mathrm{MW})\end{array}$ & $\begin{array}{c}N_{e} \\
\mathrm{HCN}\left(\mathrm{cm}^{-3}\right)\end{array}$ & $\begin{array}{c}T_{e} \\
\mathrm{ECE}(\mathrm{keV})\end{array}$ \\
\hline 79594 & 1.32 & 0.21 & $2.7 \times 10^{13}$ & 1.6 \\
79601 & 1.33 & 0.98 & $4.0 \times 10^{13}$ & 1.2 \\
79762 & 0.85 & 0.0 & $1.9 \times 10^{13}$ & 2.0 \\
79763 & 0.53 & 0.23 & $2.0 \times 10^{13}$ & 1.9 \\
79764 & 0.24 & 0.96 & $2.0 \times 10^{13}$ & 1.8 \\
79767 & 1.34 & 0.0 & $1.0 \times 10^{13}$ & 2.2 \\
79771 & 0.84 & 0.0 & $3.6 \times 10^{13}$ & 1.5 \\
79772 & 0.16 & 1.22 & $1.9 \times 10^{13}$ & 1.4 \\
\hline \hline
\end{tabular}

Lorentzian contribution from the lifetime of the lines and a Gaussian contribution from Doppler broadening are added to the instrumental profile.

\section{EXPERIMENTAL RESULTS}

\section{A. Plasma rotation and ion temperature}

As an example of plasma rotation and ion temperature measurements from the x-ray spectra we present data from a series of discharges with neutral beam injection both in the co- and counterdirection of the normal ohmic current. The data were obtained with the horizontal spectrometer. The powers of the two neutral beams were varied to produce a wide range of rotations and ion temperatures. The neutral beam powers, along with the line averaged neutral density and electron temperature, as measured from the electron cyclotron emission, are presented in Table I for these discharges. The plasma rotation and the ion temperature were obtained by fitting the spectral lines $\mathbf{w}$ and $\mathbf{z}$ to a Voigt function, which consisted of a Gaussian thermal component, a Lorentzian contribution determined from the natural line width and the above-mentioned instrumental function (see Sec. II G). The Doppler shift of the spectral lines was measured with respect to the mean zero position obtained from ohmic discharges. For this purpose, the line positions were determined from ohmic discharges for the cases when plasma current and magnetic field were in the normal directions and when the direction of both plasma current and magnetic field had been reversed. The zero velocity position was then taken to be the mean position between these two measurements. The toroidal rotation velocities in ohmic discharges, as measured from the line shifts with respect to the mean zero position, are relatively small and are about 20 $\mathrm{km} / \mathrm{s}$ in the electron drift direction.

A comparison of the measurements by x-ray spectroscopy and charge exchange recombination spectroscopy of the central toroidal plasma rotation velocities during the beam heated phase in these discharges is shown in Fig. 9. The measurements are in agreement within the error bars. The uncertainties of the x-ray measurements result from the statistical error in the determination of the line position, which is about $5-7 \mathrm{~km} / \mathrm{s}$ for these data, and from a systematic error of about $5 \mathrm{~km} / \mathrm{s}$ in determination of the zero posi- 


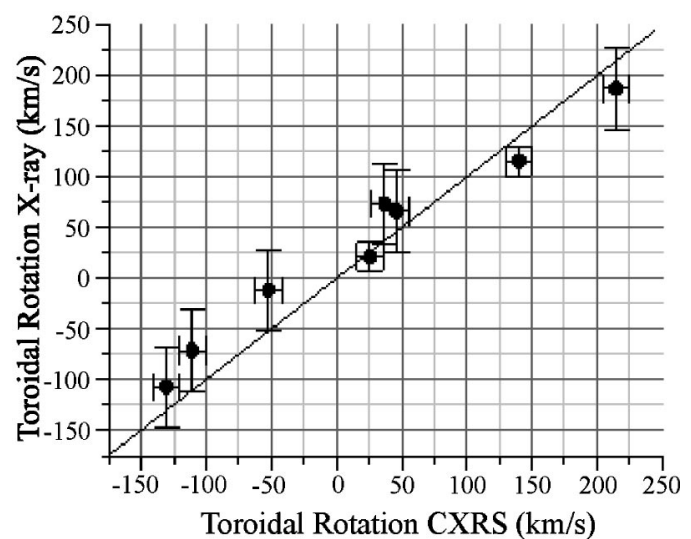

FIG. 9. Central rotation velocity on TEXTOR-94 for a series of discharges where two counterpropagating hydrogen beams were used to vary the toroidal rotation. The plot is a comparison of the measurements made by the $\mathrm{x}$-ray spectrometer to the measurements made by charge exchange recombination spectroscopy.

tion. Another systematic error is due to the fact that the x-ray measurements represent a weighted average over the radial profile of the rotation velocity. This error depends on the radial x-ray emissivity profile, which is a function of the electron temperature. An estimate for this error can be obtained for each type of discharge by plasma modeling calculations.

The primary errors for the charge-exchange spectroscopy measurements are probably due to inaccuracies in the charge exchange cross sections. ${ }^{18}$ In addition, a number of processes like level mixing from ion-ion collisions, from motional Stark and from Zeeman mixing, fine structure broadening, and the population of excited states in neutral atoms, are hard to treat theoretically and affect the accuracy of corrections to both the line position and width. It has been estimated that the total uncertainties for the charge exchange measurement will be $10 \mathrm{~km} / \mathrm{s} .{ }^{19}$ A more thorough investigation of the errors associated with the charge exchange measurements at TEXTOR-94 is planned for the future.

Figure 10 presents a comparison of measurements of the central ion temperature by $\mathrm{x}$-ray spectroscopy and charge exchange spectroscopy for the same series of discharges. The measurements are in agreement within the error bars, with the exception of the data points that refer to low-density

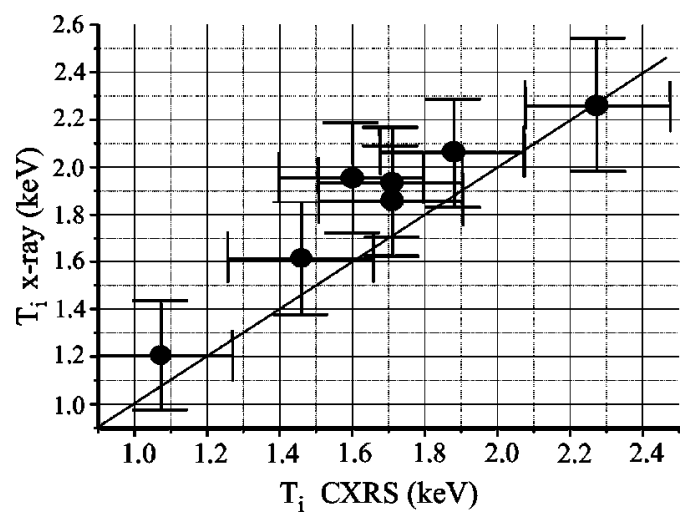

FIG. 10. A comparison between the central ion temperature measured by the $\mathrm{X}$-ray spectrometer to the ion temperature measured by CXRS. discharges. The accuracy of the x-ray measurements is affected by the statistical error of the fits, which is between 30 and $100 \mathrm{eV}$ for these discharges, and by a systematic error due to ion temperature profile effects. Ion temperature profiles were not measured for these discharges. The deviation of the line-averaged measurements from the central ion temperature will depend upon the relative width of the ion and electron temperature profiles. When the ion and electron temperature profiles are equal, as in the case of high density discharges, the central value will be underestimated by approximately $150 \mathrm{eV}$. The uncertainties, which are based on profile effects, will be resolved in the future with the imaging $\mathrm{x}$-ray crystal spectrometers that are currently under development. The error of the charge exchange measurements was estimated to be $200 \mathrm{eV}$. It results from the same problems previously mentioned for the rotation measurements.

\section{B. Electron temperature}

The electron temperature can be obtained from the intensity ratio of the dielectronic satellite $k$ to the resonance line w. However, since the spectrometer integrates over a line of sight through the plasma, care must be taken to correctly infer the value of the central electron temperature from these $\mathrm{x}$-ray measurements. In order to obtain a reliable value for the central electron temperature, the observed spectra have been compared with simulated line-averaged spectra from theoretical data. The synthetic spectra included direct electron impact excitation from the ground state to $n \geqslant 2$ with cascades, radiative recombination, dielectronic recombination, inner-shell excitation, and inner-shell ionization. The intensities of the spectral lines were calculated for each radial position $r$ from

$$
I(r)=n_{e}(r)^{*} n_{\text {ion }}\left(T_{e}(r)\right)^{*}\left\langle\sigma v\left(T_{e}(r)\right)\right\rangle \text { line, process, }
$$

where $n_{e}(r)$ is the electron density, $n_{\text {ion }}(r)$ is the density of the charge state from which the line originates, and $\left\langle\sigma v\left(T_{e}(r)\right)\right\rangle_{\text {line }}$ is the excitation rate for of the spectral line for a particular process. The spectra were then integrated over the line of sight using electron temperature profiles measured by ECE, density profiles from the scaling with the safety factor $q$ according to Schueller et al. ${ }^{20}$ and assuming coronal equilibrium for the charge state distribution. The excitation rates which were used to fit the $\mathbf{w}, \mathbf{x}, \mathbf{y}$, and $\mathbf{z}$ lines were obtained by an extrapolation of the data for calcium calculated by Bely-Dubau et al. ${ }^{21}$ to argon according to Mewe et $^{2}$ l. $^{22,23}$ The wavelengths and satellite intensities were calculated by Vainsthein et $_{\text {al. }}{ }^{24,25}$

Figure 11 shows a comparison of the central electron temperature measured by $\mathrm{x}$ rays to that measured by ECE for a series of discharges in hydrogen, deuterium, and helium with electron densities ranging from $1.0 \times 10^{13}-4.0$ $\times 10^{13} \mathrm{~cm}^{-3}$ and central electron temperatures ranging from 1.0 to $2.4 \mathrm{keV}$. The agreement between the two diagnostics is remarkably good for a wide range of plasma conditions in TEXTOR-94. The error bar shown in Fig. 11 represents only the error from the least squares fit of the synthetic spectra. The errors which can result from uncertainties of the atomic data will be addressed in a forthcoming article. The error in 


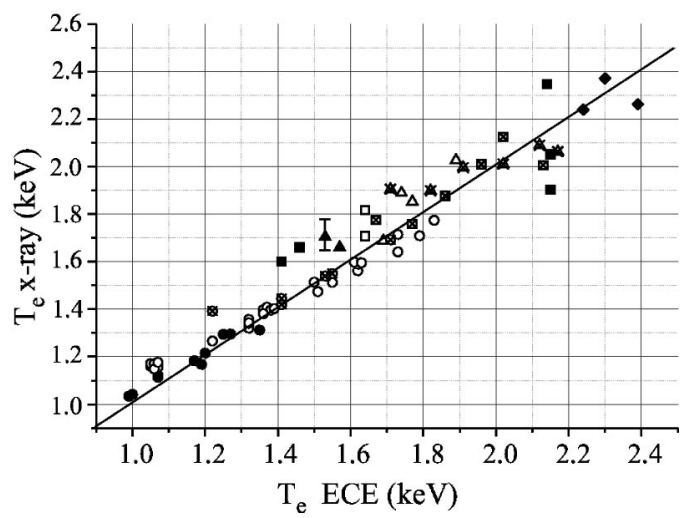

FIG. 11. Comparison of the electron temperature measured by the $\mathrm{x}$-ray spectrometer and the ECE diagnostic for a series of discharges with hydrogen (solid), deuterium (open), and helium (open with cross) as working gases. In this series of discharges, the electron densities ranged from 1.0 $\times 10^{13}$ to $4.0 \times 10^{13} \mathrm{~cm}^{-3}$. The circles are ohmic discharges, the squares with beam 1 hydrogen injection, the triangles with beam 2 hydrogen injection, and the diamonds with both beam 1 and beam 2 hydrogen injection.

the ECE measurements is estimated to be less than $20 \%{ }^{26}$ based on a recent calibration of the instrument.

\section{Measurements of ArXVI/ArXVII}

The relative abundance of ArXVI/ArXVII can be determined from the ratio of the satellites, $1 s^{2} 2 s-1 s 2 p 2 s$, which are produced from the lithium-like ground state by collisional inner-shell excitation, to the helium-like resonance line, $1 s^{2}-1 s 2 p$. For this purpose, the experimental spectra have been fitted by simulated line-averaged spectra in the manner described above. Figure 12 shows the value of ArXVI/ArXVII, which was obtained from the fit, for ohmic deuterium discharges, both with and without the injection of a neutral hydrogen beam as a function of density. The observed values of ArXVI/ArXVII have been normalized to those predicted by coronal equilibrium. The observed values of ArXVI/ArXVII are always larger than the predictions from coronal equilibrium. These deviations from coronal equilibrium are too large to be ascribed to theoretical uncertainties in the coronal equilibrium data. We also point out

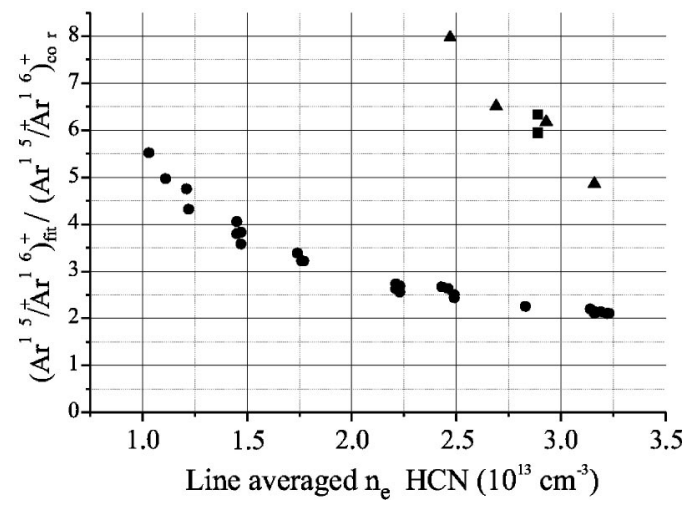

FIG. 12. Comparison of the central ArXVI/ArXVII ratio obtained from a fit of the experimental spectra to the value predicted by coronal equilibrium as a function of the line averaged density. The circles are ohmic, squares have beam 1 hydrogen injection, and up triangles have beam 2 hydrogen injection. The data come from a series of deuterium discharges from TEXTOR shot $81150-81161$.

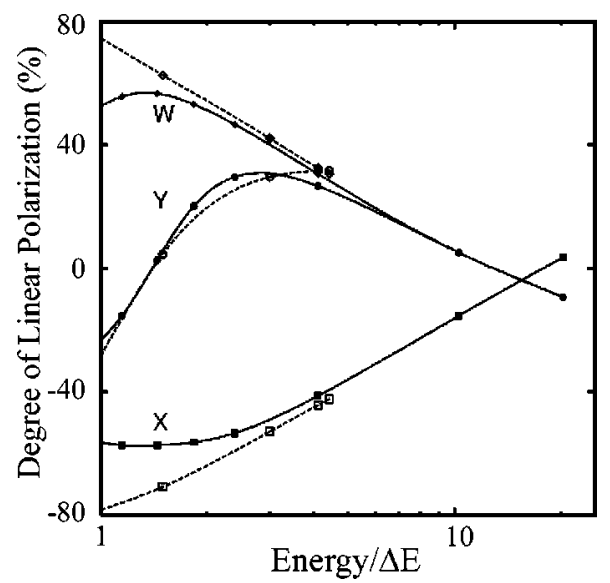

FIG. 13. Calculated (Ref. 27) polarization of the $w, x$, and $y$ lines of the ArXVII spectrum predicted for a monoenergetic electron beam.

that coronal equilibrium is independent of the electron density and only a function of the electron temperature. The strong dependence of ArXVI/ArXVII on electron density suggests that the charge state distribution is also determined by diffusion and charge exchange. A detailed analysis of the data with respect to these processes has been presented in Ref. 13.

\section{Polarization during ohmic discharges}

Polarization of line radiation can be produced by an anisotropy of the electron energy distribution. A beam component in the electron energy distribution along the toroidal direction can be expected in tokamak plasmas under some operating conditions. This can lead to polarization, if the nonthermal electrons fall into the right energy range. ${ }^{27,28}$ Figure 13 shows polarization effects for the prominent lines of the ArXVII spectrum. The results were obtained from modeling calculations, which assume a beam of monoenergetic electrons in the energy range from 3.2 to $136 \mathrm{keV} .{ }^{27}$ If there is a beam component along the toroidal direction, then the relative intensity between two of the polarized lines $(\mathbf{w}, \mathbf{x}$, and $\mathbf{y}$ ) in the spectrum will be different for the two states of polarization. A comparison of the line ratios in the two spectrometers is therefore a measure of the degree of polarization of the radiation, and a measure of the nonthermal component in the electron distribution function. The observed polarization will strongly depend on the amount of beam electrons relative to thermal electrons.

One might expect that the polarization of the spectrum is most pronounced in normal discharges with low temperatures where the ratio of nonthermal to thermal electrons with energies higher than the threshold for collisional excitation would be largest. To look for polarization under these conditions, ohmically heated discharges with low electron temperatures were investigated during two phases with different plasma current. During the first phase, the current, lineaveraged electron density and electron temperature were 210 $\mathrm{kA}, 2.0 \times 10^{13} \mathrm{~cm}^{-3}$, and $1.0 \mathrm{keV}$, respectively. During the second phase, the current was $350 \mathrm{kA}$, the line-averaged electron density was $2.5 \times 10^{13} \mathrm{~cm}^{-3}$, and the electron temperature was $1.1 \mathrm{keV}$. Due to the geometry of the vertical 
TABLE II. Line intensity ratios for two ohmic conditions in both the horizontal and vertical spectrometer. Since the line ratios in both systems are the same within the accuracy of the measurement, no polarization was observed.

\begin{tabular}{ccc}
\hline \hline Line intensity ratio & Horizontal spectrometer & Vertical spectrometer \\
\hline Plasma current $=210 \mathrm{kA}$ & \multicolumn{2}{c}{$N_{e}=2.0 \times 10^{13} \mathrm{~cm}^{-3}$} \\
$x / w$ & 0.16 & 0.16 \\
$y / w$ & 0.24 & 0.23 \\
$z / w$ & 0.54 & 0.55 \\
& \multicolumn{2}{c}{$N_{e}=2.5 \times 10^{13} \mathrm{~cm}^{-3}$} \\
Plasma current $=350 \mathrm{kA}$ & 0.15 & 0.16 \\
$x / w$ & 0.23 & 0.24 \\
$y / w$ & 0.52 & 0.56 \\
$z / w$ & & \\
\hline \hline
\end{tabular}

spectrometer, each spectral line originates from a slightly different radial position in the plasma. To guarantee that the spectral lines seen by both spectrometers came from the same plasma volume, a series of discharges were required to measure the emission profiles of the lines, by rotating the crystal in the vertical spectrometer. It was found that small variations in the position and width of the emission profiles can change the line ratios drastically in the vertical system. ${ }^{29}$ The line ratios for the two phases, corrected for profile effects as described in Ref. 28, are given in Table II for both spectrometers. Since the ratios are equal to within the errors of the measurements, it can be concluded that, within the sensitivity of the instrument, no polarization of the radiation was found. Other promising operating conditions for the polarization measurements are under investigation. At lower densities, in the slide-away regime of the tokamak, polarization by a direct beam component might be more easily observed due to the larger beam component along the toroidal direction. Also, during the fast crash of the sawtooth oscillations, the electron distribution function can be expected to change significantly, possibly leading to polarized line radiation.

\section{ACKNOWLEDGMENTS}

The authors would like to thank R. Jaspers for providing the ion temperature and toroidal rotation from charge exchange spectroscopy, R. Koslowski for providing the neutral density measurements, and A. Krämer-Flecken for providing the electron temperature measurements from the ECE diagnostic. The authors would also like to express their thanks to the TEXTOR-94 team, with additional thanks to G. Telemann and A. Scholl for direct support of the diagnostic. The multiwire proportional detectors and part of the detection electronics used in the present $\mathrm{x}$-ray polarimeter are supplied by the Princeton Plasma Physics Laboratory via loan agreement PPPL 94-18-03. Two authors (J. W. and I. A.) gratefully acknowledge the financial assistance from the Graduiertenkolleg.
${ }^{1}$ G. Bertschinger, W. Biel, TEXTOR-94 team, O. Herzog, J. Weinheimer, H.-J. Kunze, and M. Bitter, Phys. Scr., T 83, 132 (1999).

${ }^{2}$ T. Fujimoto and S. Kazantsev, Plasma Phys. Controlled Fusion 37, A135 (1995).

${ }^{3}$ M. Bitter, S. von Goeler, R. Horton, M. Goldman, K. W. Hill, N. R. Sauthoff, and W. Stodiek, Phys. Rev. Lett. 42, 304 (1979).

${ }^{4}$ M. Bitter et al., Phys. Rev. Lett. 43, 129 (1979).

${ }^{5}$ J. Dubau and M. Loulergue, J. Phys. B 15, 1007 (1982).

${ }^{6}$ K.-D. Zastrow, E. Källne, and H. P. Summers, Phys. Rev. A 41, 1427 (1990).

${ }^{7}$ W. Biel, G. Bertschinger, R. Dux, R. Jaspers, H. R. Koslowski, A. Krämer-Flecken, M. Lehnen, J. Rapp, B. Unterberg, J. Weinheimer, and TEXTOR team, 26th Eur. Phys. Soc. Conf. Controlled Fusion Plasma Physics, Maastricht, poster 2P.017, June 1999.

${ }^{8}$ TFR Group, F. Bombarda, F. Bely-Dubau, P. Faucher, M. Cornille, J. Dubau, and M. Loulergue, Phys. Rev. A 32, 2374 (1985).

${ }^{9}$ P. Platz, M. Cornille, and J. Dubau, J. Phys. B: At. Mol. Opt. Phys. 29, 3787 (1996).

${ }^{10}$ J. E. Rice, E. S. Marmar, E. Källne, and J. Källne, Phys. Rev. A 35, 3033 (1987).

${ }^{11}$ M. Bitter, G. Bertschinger, W. Biel, G. Fuchs, S. von Goeler, H.-J. Kunze, D. Rusbüldt, and J. Weinheimer, 26th Eur. Phys. Soc. Conf. Controlled Fusion Plasma Physics, Maastricht, poster 4P.084, June 1999.

${ }^{12}$ K-D Zastrow et al., 26th Eur. Phys. Soc. Conf. Controlled Fusion Plasma Physics, Maastricht, poster 1P.028, June 1999.

${ }^{13}$ J. Weinheimer, W. Biel, G. Bertschinger, M. Bitter, and H.-J. Kunze, 26th Eur. Phys. Soc. Conf. Controlled Fusion Plasma Physics, Maastricht, poster 2P.014, June 1999.

${ }^{14}$ R. A. Boie, J. Fischer, and V. Radeka, Nucl. Instrum. Methods Phys. Res. 201, 93 (1982).

${ }^{15}$ K. W. Hill et al., Rev. Sci. Instrum. 56, 1165 (1985).

${ }^{16}$ G. Borchert et al., Nucl. Instrum. Methods Phys. Res. Sect. A (to be published).

${ }^{17}$ I. Uschmann, E. Förster, K. Gäbel, G. Hölzer, and M. Ensslen, J. Appl. Crystallogr. 26, 405 (1993).

${ }^{18}$ R. B. Howell, R. J. Fonck, R. J. Knize, and K. P. Jaehnig, Rev. Sci. Instrum. 59, 1521 (1988).

${ }^{19}$ R. Jaspers, personal communication.

${ }^{20}$ F. Schueller, D. Schram, J. Konings, A. van Lammeren, J. Timmermans, M. Vereck, and TRP-team, 18th European Conference on Controlled Fusion and Plasma Physics, Berlin, IV-185-188, 3-7 June 1991.

${ }^{21}$ F. Bely-Dubau, J. Dubau, P. Faucher, A. H. Gabriel, M. Loulergue, L. Steenman-Clark, S. Volonte, E. Antonucci, and C. G. Rapley, Mon. Not. R. Astron. Soc. 201, 1155 (1982).

${ }^{22}$ R. Mewe, J. Schrijver, and J. Sylvester, Astron. Astrophys. 87, 55 (1980).

${ }^{23}$ R. Mewe, J. Schrijver, and J. Sylvester, Astron. Astrophys., Suppl. Ser. 40, 323 (1980).

${ }^{24}$ L. A. Vainstein and U. I. Safronova, At. Data Nucl. Data Tables 21, 49 (1978).

${ }^{25}$ L. A. Vainstein, U. I. Safronova, and P. N. Lebedev, Institute of Spectroscopy, Report No. 2, 1985.

${ }^{26}$ A. Krämer-Flecken, personal communication.

${ }^{27}$ A. S. Schlyaptseva, A. M. Urnov, A. Vinogradov, "Polarization of Lines of Multi-Charged Ions excited from Electron Impact,' P. N. Lebedev Institute Rep. 194, 1981.

${ }^{28}$ M. K. Inal, J. Dubau, J. Phys. B: At. Mol. Phys. 20, 4221 (1987).

${ }^{29}$ J. Weinheimer, O. Herzog, G. Bertschinger, M. Bitter, and H.-J. Kunze, ICCP and 25th EPS Controlled Fusion Plasma Physics, Prague, poster p2.096, June/July 1998. 